\title{
Correction: Bushen-Yizhi formula ameliorates cognitive dysfunction through SIRT1/ER stress pathway in SAMP8 mice
}

\author{
Shi-Jie Zhang ${ }^{1, *}$, Ting-Ting $X \mathbf{u}^{1,}{ }^{*}$, Lin $\mathrm{Li}^{1}$, Yu-Min $X \mathbf{u}^{1}$, Zi-Ling Qu ${ }^{1}$, Xin-Chen Wang ${ }^{1}$,

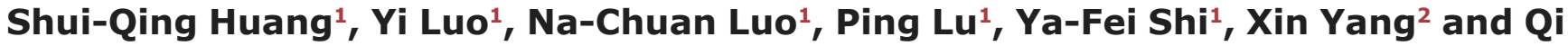 \\ Wang $^{1}$ \\ ${ }^{1}$ Institute of Clinical Pharmacology, Guangzhou University of Chinese Medicine, Guangzhou 510405, China \\ ${ }^{2}$ Department of Pharmacy, The Fifth Affiliated Hospital of Guangzhou Medical University, Guangzhou 510700, China \\ *These authors contributed equally to this work
}

Published: July 07, 2020

Copyright: Zhang et al. This is an open-access article distributed under the terms of the Creative Commons Attribution License 3.0 (CC BY 3.0), which permits unrestricted use, distribution, and reproduction in any medium, provided the original author and source are credited.

This article has been corrected: While reviewing the database for this study, the authors discovered that the Tunel staining data for all 6 panels in Figure 4C did not represent the relevant group. The corrected Figure 4, obtained using original data, is given below. The authors declare that these corrections do not change the results or conclusions of this paper.

Original article: Oncotarget. 2017; 8:49338-49350. https://doi.org/10.18632/oncotarget.17638 

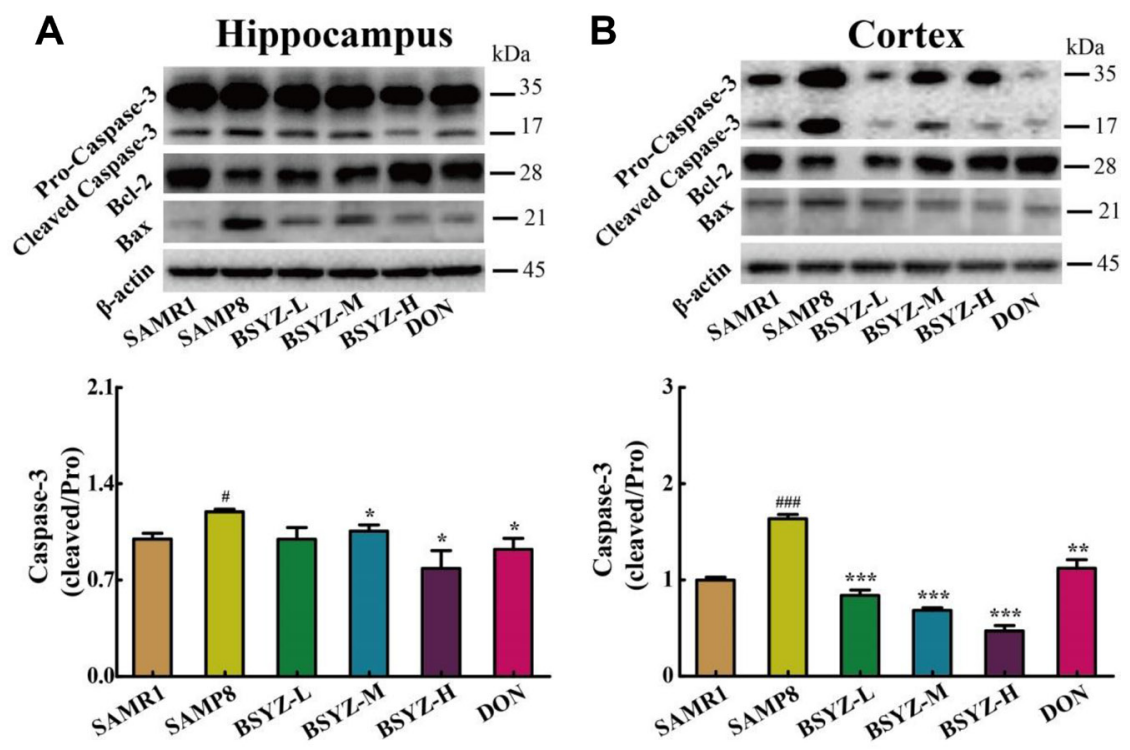

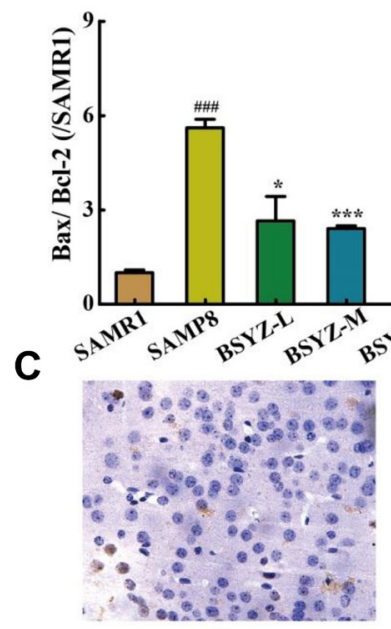

SAMR1

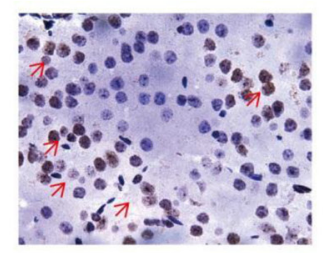

BSYZ-M

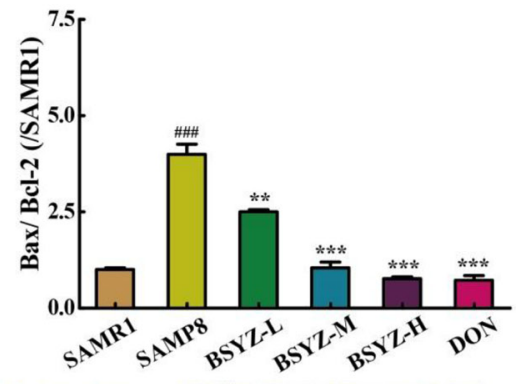

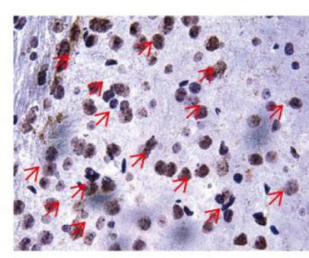

SAMP8

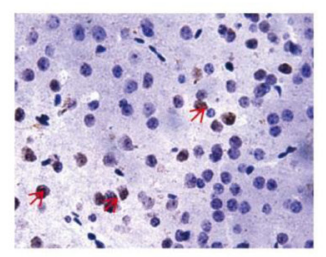

BSYZ-H

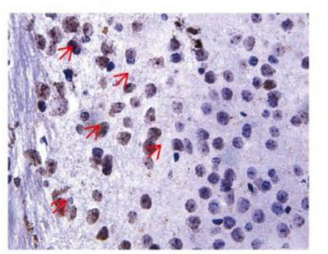

BSYZ-L

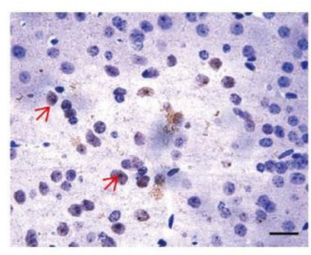

DON

Figure 4: BSYZ protects against apoptosis in both hippocampus and cortex. Western blot analysis of Bax, Bcl-2 and Caspase-3 in hippocampus (A) and cortex (B). (C) TUNEL staining in parietal cortex in mice. Scale bar: $50 \mu \mathrm{m}$. BSYZ-L: Bushen-Yizhi (1.46 g/kg/d); BSYZ-M: Bushen-Yizhi $(2.92 \mathrm{~g} / \mathrm{kg} / \mathrm{d})$; BSYZ-H: Bushen-Yizhi $(5.84 \mathrm{~g} / \mathrm{kg} / \mathrm{d})$; DON: donepezil. Data represent mean $\pm \mathrm{SEM}(n=20 \mathrm{per}$ group). ${ }^{\#} P<0.05,{ }^{\# \# \#} P<0.001$ vs. SAMR1; ${ }^{*} P<0.05,{ }^{* *} P<0.01,{ }^{* * *} P<0.001$ vs. SAMP8. 